\title{
Trust-Region-Based Convergence Safeguards for Space Mapping Design Optimization of Microwave Circuits
}

\author{
Slawomir Koziel ${ }^{\ddagger 1}$, John W. Bandler ${ }^{\dagger \diamond 2}$, and Qingsha S. Cheng ${ }^{\dagger 3}$ \\ $\$$ School of Science and Engineering, Reykjavik University, Kringlunni 1, IS-103 Reykjavik, Iceland \\ ${ }^{\dagger}$ Department of Electrical \& Computer Engineering, McMaster University, Hamilton, ON, Canada L8S 4K1 \\ ${ }^{\diamond}$ Bandler Corporation, Dundas, ON, Canada L9H 5E7 \\ ${ }^{1}$ koziel@ru.is, ${ }^{2}$ bandler@mcmaster.ca, ${ }^{3}$ chengq@mcmaster.ca
}

\begin{abstract}
Convergence is a well-known issue for space mapping (SM) optimization algorithms. One possible convergence safeguard is the trust region (TR) approach where the surrogate model is optimized in a restricted neighborhood of the current iteration point. We demonstrate that although formal conditions for applying trust regions are not strictly satisfied for SM surrogate models, TR improves the stability and convergence properties of the SM optimization process. Further improvement can be realized when approximate fine model Jacobian information is exploited in the construction of the SM surrogate.

Index Terms-Computer-aided design (CAD), EM optimization, space mapping, trust-region methods.
\end{abstract}

\section{INTRODUCTION}

Space mapping (SM) has proven to be one of the most efficient design optimization methodologies in microwave engineering to date [1]-[4]. It avoids direct optimization of a CPU-intensive "fine" model by shifting the optimization burden to a cheap and physically-based "coarse" model.

A serious issue for SM algorithms is convergence, which depends on the similarity between the fine model and the SM surrogate [2]. Trust-region (TR) [5] is one of the possible approaches that can be used to amend the convergence properties of SM algorithms. Formally, using TR with space mapping is not correct because the first order consistency [6] between the fine model and SM surrogate does not usually hold. Still, as observed, TR may work well with SM algorithms [4].

Here, we present a systematic treatment of TR-enhanced SM algorithms, give a heuristic explanation of why TR actually works with SM, as well as propose a modification of the TR-SM algorithm, which uses an approximate fine model Jacobian to enhance the surrogate model. Several microwave design problems verify our work.

\section{SPACE MAPPING With TR CONVERGENCE SAFEGUARDS}

\section{A. Standard SM Algorithm}

The microwave design optimization problem is defined as

$$
\boldsymbol{x}_{f}^{*}=\arg \min _{\boldsymbol{x}} U\left(\boldsymbol{R}_{f}(\boldsymbol{x})\right)
$$

This work was supported in part by the Natural Sciences and Engineering Research Council of Canada under Grants RGPIN7239-06, STPGP33676006, and by Bandler Corporation. where $\boldsymbol{R}_{f}: X_{f} \rightarrow R^{m}, X_{f} \subseteq R^{n}$, denotes the response vector of a fine model of the device of interest; $U: R^{m} \rightarrow R$ is a given objective function, e.g., minimax [1].

Direct optimization of the fine model is replaced by an iterative procedure generating a sequence of points $\boldsymbol{x}^{(i)} \in X_{f}$, $i=0,1,2, \ldots$, and a family of surrogate models $\boldsymbol{R}_{s}^{(i)}: X_{s}^{(i)} \rightarrow R^{m}, X_{s}^{(i)} \subseteq R^{n}, i=0,1, \ldots$, so that

$$
\boldsymbol{x}^{(i+1)}=\arg \min _{\boldsymbol{x}} U\left(\boldsymbol{R}_{s}^{(i)}(\boldsymbol{x})\right)
$$

Let $\boldsymbol{R}_{c}: X_{c} \rightarrow R^{m}, X_{c} \subseteq R^{n}$, denote the response vectors of the coarse model that describes the same object as the fine model: less accurate but much faster to evaluate. Surrogate models $\boldsymbol{R}_{s}^{(i)}$ in (2) are constructed as follows:

$$
\boldsymbol{R}_{s}^{(i)}(\boldsymbol{x})=\overline{\boldsymbol{R}}_{s}\left(\boldsymbol{x}, \boldsymbol{p}^{(i)}\right)
$$

where $\overline{\boldsymbol{R}}_{s}: X_{s} \rightarrow R^{m}$ is a generic SM surrogate model which is $\boldsymbol{R}_{c}$ composed with some suitable SM transformations, and $X_{s} \subseteq X_{c} \times X_{p}$, with $X_{p}$ being the parameter space of these transformations. A vector of SM parameters, $\boldsymbol{p}^{(i)}$, is obtained using the parameter extraction procedure

$$
\boldsymbol{p}^{(i)}=\arg \min _{\boldsymbol{p}} \sum_{k=0}^{i}\left\|\boldsymbol{R}_{f}\left(\boldsymbol{x}^{(k)}\right)-\overline{\boldsymbol{R}}_{s}\left(\boldsymbol{x}^{(k)}, \boldsymbol{p}\right)\right\|
$$

An example of a generic SM model is an input SM of the form $\boldsymbol{x} \rightarrow \boldsymbol{B} \boldsymbol{x}+\boldsymbol{c}$, where $\overline{\boldsymbol{R}}_{s}(\boldsymbol{x}, \boldsymbol{p})=\overline{\boldsymbol{R}}_{s}(\boldsymbol{x}, \boldsymbol{B}, \boldsymbol{c})=\boldsymbol{R}_{c}(\boldsymbol{B} \cdot \boldsymbol{x}+\boldsymbol{c})$. A variety of other SM surrogates can be found in [1], [2], [4].

As the algorithm (2)-(4) accepts a new design $\boldsymbol{x}^{(i+1)}$ regardless of the $\boldsymbol{R}_{f}$ specification error improvement, convergence of the SM algorithm is not guaranteed [2]. Moreover, as the perfect matching between the $\boldsymbol{R}_{s}^{(i)}$ and $\boldsymbol{R}_{f}$ at $\boldsymbol{x}^{(i)}$ is not ensured, there is no guarantee for the SM algorithm to locate the fine model optimal solution [2]. Excellent results reported in the literature [1]-[4], obtained with SM, are due to carefully chosen coarse models and properly selected SM type.

\section{B. Trust-Region Enhanced SM Algorithm}

A trust region (TR) approach [5] can be used to improve the convergence properties of the SM algorithm. In particular, the surrogate optimization process (2) can be constrained to a neighborhood of $\boldsymbol{x}^{(i)}$, defined as $\left\|\boldsymbol{x}-\boldsymbol{x}^{(i)}\right\| \leq \delta^{(i)}$, as follows 


$$
\boldsymbol{x}^{(i+1)}=\arg \min _{\boldsymbol{x},\left\|\boldsymbol{x}-\boldsymbol{x}^{(i)}\right\|<\delta^{(i)}} U\left(\boldsymbol{R}_{s}^{(i)}(\boldsymbol{x})\right)
$$

where $\delta^{(i)}$ is a TR radius at iteration $i$. The TR radius is reduced if the improvement of the fine model objective function is not sufficient, i.e., if $\left(U\left(\boldsymbol{R}_{f}\left(\boldsymbol{x}^{(i+1)}\right)\right)-U\left(\boldsymbol{R}_{f}\left(\boldsymbol{x}^{(i)}\right)\right)\right) /$ $\left(U\left(\boldsymbol{R}_{s}^{(i)}\left(\boldsymbol{x}^{(i+1)}\right)\right)-U\left(\boldsymbol{R}_{s}^{(i)}\left(\boldsymbol{x}^{(i)}\right)\right)\right)$ is too small, or if $U\left(\boldsymbol{R}_{f}\left(\boldsymbol{x}^{(i+1)}\right)\right) \geq U\left(\boldsymbol{R}_{f}\left(\boldsymbol{x}^{(i)}\right)\right)$, in which case the new design is rejected. We use the standard TR radius updating rules [5].

If, for all $i=0,1,2, \ldots$, the surrogate model satisfies the zero- and first-order consistency conditions of the form

$$
\begin{aligned}
\boldsymbol{R}_{s}^{(i)}\left(\boldsymbol{x}^{(i)}\right) & =\boldsymbol{R}_{f}\left(\boldsymbol{x}^{(i)}\right) \\
\boldsymbol{J}_{\boldsymbol{R}_{s}^{(i)}}\left(\boldsymbol{x}^{(i)}\right) & =\boldsymbol{J}_{\boldsymbol{R}_{f}}\left(\boldsymbol{x}^{(i)}\right)
\end{aligned}
$$

where $\boldsymbol{J}$ denotes the Jacobian of the respective model, then, under mild assumptions concerning smoothness of the models, algorithm (5) is convergent to the local fine model optimum [7]. The fundamental reason is that (6) and (7) ensure that $U\left(\boldsymbol{R}_{f}\left(\boldsymbol{x}^{(i+1)}\right)\right)<U\left(\boldsymbol{R}_{f}\left(\boldsymbol{x}^{(i)}\right)\right)$ if the TR radius is sufficiently small.

Unfortunately, (6) and (7) are not necessarily satisfied by the SM surrogate model. In particular, the SM optimization process may get stuck at some point as the reduction of the TR radius $\delta^{(i)}$ does not bring any improvement of the fine model objective function, which results in the termination of the algorithm.

On the other hand, an important prerequisite of SM is that $\boldsymbol{R}_{c}$ is physically-based so that the surrogate model reflects the general features of $\boldsymbol{R}_{f}$; in particular, the local behavior of both models is similar. This, in combination with the multi-point parameter extraction (4) ensures that (6) and (7) may be satisfied approximately. Moreover, condition (6) can be easily enforced by means of the output SM [1] using the surrogate

$$
\boldsymbol{R}_{s}^{(i)}(\boldsymbol{x})=\overline{\boldsymbol{R}}_{s}\left(\boldsymbol{x}, \boldsymbol{p}^{(i)}\right)+\boldsymbol{d}^{(i)}
$$

with $\boldsymbol{p}^{(i)}$ obtained by (4) and

$$
\boldsymbol{d}^{(i)}=\boldsymbol{R}_{f}\left(\boldsymbol{x}^{(i)}\right)-\overline{\boldsymbol{R}}_{s}\left(\boldsymbol{x}^{(i)}, \boldsymbol{p}^{(i)}\right)
$$

Numerical results presented in Section III demonstrate that a TR-enhanced SM algorithm with output SM (8) and (9) exhibits improvement over the standard SM algorithm.

\section{Improved Trust-Region Enhanced SM Algorithm}

The SM algorithm can be further improved if condition (7) is satisfied. This can be realized with the following model:

$$
\boldsymbol{R}_{s}^{(i)}(\boldsymbol{x})=\overline{\boldsymbol{R}}_{s}\left(\boldsymbol{x}, \boldsymbol{p}^{(i)}\right)+\boldsymbol{d}^{(i)}+\boldsymbol{E}^{(i)}\left(\boldsymbol{x}-\boldsymbol{x}^{(i)}\right)
$$

where SM parameters $\boldsymbol{p}^{(i)}$ are obtained with (4), vector $\boldsymbol{d}^{(i)}$ is computed according to (8), whereas

$$
\boldsymbol{E}^{(i)}=\boldsymbol{J}_{\boldsymbol{R}_{f}}\left(\boldsymbol{x}^{(i)}\right)-\boldsymbol{J}_{\overline{\boldsymbol{R}}_{s}\left(\cdot, p^{(i)}\right)}\left(\boldsymbol{x}^{(i)}\right)
$$

Model (11) explicitly uses $\boldsymbol{R}_{f}$ sensitivity information, which is computationally prohibitive if the Jacobian is to be evaluated using finite differences. Another option is adjoint sensitivity [8], but it is not yet commercially available.

Here, we exploit an approximation of the term $\boldsymbol{E}^{(i)}$ obtained using a Broyden update [9]. Jacobians of $\boldsymbol{R}_{f}$ and the SM surrogate are estimated jointly as follows:

$$
\boldsymbol{E}^{(i)}=\boldsymbol{J}_{\boldsymbol{B} \cdot \boldsymbol{R}_{f}-\overline{\boldsymbol{R}}_{s}}^{(i)}
$$

where
$\boldsymbol{J}_{\boldsymbol{B} \cdot \boldsymbol{R}_{f}-\bar{R}_{s}}^{(i)}=\boldsymbol{J}_{\boldsymbol{B} \cdot \boldsymbol{R}_{f}-\bar{R}_{s}}^{(i-1)}+\frac{\left(\overline{\boldsymbol{f}}^{(i)}-\boldsymbol{J}_{\boldsymbol{B} \cdot \boldsymbol{R}_{f}-\bar{R}_{s}}^{(i-1)} \cdot \boldsymbol{h}^{(i)}\right) \cdot \boldsymbol{h}^{(i) T}}{\boldsymbol{h}^{(i) T} \boldsymbol{h}^{(i)}}, i=1,2, \ldots$

with $\overline{\boldsymbol{f}}^{(i)}=\left(\boldsymbol{R}_{f}\left(\boldsymbol{x}^{(i)}\right)-\overline{\boldsymbol{R}}_{s}\left(\boldsymbol{x}^{(i)}, \boldsymbol{p}^{(i)}\right)\right)-\left(\boldsymbol{R}_{f}\left(\boldsymbol{x}^{(i-1)}\right)-\overline{\boldsymbol{R}}_{s}\left(\boldsymbol{x}^{(i-1)}, \boldsymbol{p}^{(i)}\right)\right)$, $\boldsymbol{h}^{(i)}=\boldsymbol{x}^{(i)}-\boldsymbol{x}^{(i-1)}$, and $\boldsymbol{J}_{\boldsymbol{B} \cdot \boldsymbol{R}_{f}-\overline{\boldsymbol{R}}_{s}}^{(0)}=\mathbf{0}_{m \times n}$.

To improve accuracy of estimate (12) we consider local updates using only points satisfying $\left\|\boldsymbol{x}^{(k)}-\boldsymbol{x}^{(i)}\right\| \leq C$, where $C$ is a user-defined threshold value (typically 0.1 ).

Obviously, the $\boldsymbol{E}$ term calculated using (12) and (13) does not guarantee the exact satisfaction of (7), however, it can be shown that the model (10)-(13) ensures the fundamental property $U\left(\boldsymbol{R}_{f}\left(\boldsymbol{x}^{(i+1)}\right)\right)<U\left(\boldsymbol{R}_{f}\left(\boldsymbol{x}^{(i)}\right)\right)$ for sufficiently small TR radius assuming smoothness of the functions involved.

\section{VERIFICATION EXAMPLES}

\section{A. Test Problem Formulation}

Problem 1: Seven-section capacitively-loaded impedance transformer [10]. Both "coarse" and "fine" models (Fig. 1) are implemented in Matlab. The design parameters are

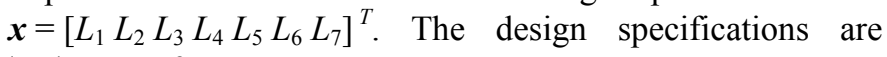
$\left|S_{11}\right| \leq 0.07$ for $1.0 \mathrm{GHz} \leq \omega \leq 7.7 \mathrm{GHz}$.

Problem 2: Second-order capacitively-coupled dualbehavior resonator microstrip filter [11] (Fig. 2). The design parameters are $\boldsymbol{x}=\left[\begin{array}{lll}L_{1} & L_{2} & L_{3} S\end{array}\right]^{T}$. The fine model is simulated in FEKO [12]. The coarse model, Fig. 3, is implemented in Agilent ADS [13]. The design specifications are $\left|S_{21}\right| \geq-3 \mathrm{~dB}$ for $3.8 \mathrm{GHz} \leq \omega \leq 4.2 \mathrm{GHz}$, and $\left|S_{21}\right| \leq-20 \mathrm{~dB}$ for $2.0 \mathrm{GHz} \leq \omega \leq 3.2 \mathrm{GHz}$ and $4.8 \mathrm{GHz} \leq \omega \leq 6.0 \mathrm{GHz}$.

Problem 3: Third-order Chebyshev bandpass filter [14] (Fig. 4). The design parameters are $\boldsymbol{x}=\left[L_{1} L_{2} S_{1} S_{2}\right]^{T} \mathrm{~mm}$. Other parameters are: $W_{1}=W_{2}=0.4 \mathrm{~mm}$. The fine model $\boldsymbol{R}_{f}$ is simulated in Sonnet $\boldsymbol{e m}$ [15]. The coarse model, Fig. 5, is implemented in Agilent ADS [13]. The design specifications are $\left|S_{21}\right| \geq-3 \mathrm{~dB}$ for $1.8 \mathrm{GHz} \leq \omega \leq 2.2 \mathrm{GHz}$, and $\left|S_{21}\right| \leq-$ $20 \mathrm{~dB}$ for $1.0 \mathrm{GHz} \leq \omega \leq 1.6 \mathrm{GHz}$ and $2.4 \mathrm{GHz} \leq \omega \leq 3.0 \mathrm{GHz}$.

Problem 4: Open-loop ring resonator bandpass filter [16] (Fig. 6). The design parameters are $\boldsymbol{x}=\left[\begin{array}{llllll}L_{1} & L_{2} & L_{3} & L_{4} & S_{1} & S_{2}\end{array}\right]^{T}$ $\mathrm{mm}$. Other parameter values are: $W=0.6 \mathrm{~mm}, W_{1}=0.4 \mathrm{~mm}$. $\boldsymbol{R}_{f}$ is simulated in FEKO [12]. The coarse model (Fig. 7) is implemented in Agilent ADS [13]. The design specifications are $\left|S_{21}\right| \geq-3 \mathrm{~dB}$ for $2.8 \mathrm{GHz} \leq \omega \leq 3.2 \mathrm{GHz}$, and $\left|S_{21}\right| \leq-20$ $\mathrm{dB}$ for $1.5 \mathrm{GHz} \leq \omega \leq 2.5 \mathrm{GHz}$ and $3.5 \mathrm{GHz} \leq \omega \leq 4.5 \mathrm{GHz}$.

\section{B. Experimental Setup}

For our four test problems we compared performance of the

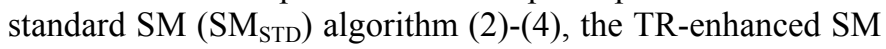
$\left(\mathrm{SM}_{\mathrm{TR}}\right)$ algorithm (5) with the output SM model (8), (9), and the TR-enhanced SM ( $\mathrm{SM}_{\mathrm{TR}-\mathrm{B}}$ ) algorithm using model (10)(13). In all cases the algorithm was terminated if one of the following conditions was satisfied: $\left\|\boldsymbol{x}^{(i+1)}-\boldsymbol{x}^{(i)}\right\|<10^{-2}$, $\delta^{(i+1)}<10^{-3}$, or $U\left(\boldsymbol{R}_{f}\left(\boldsymbol{x}^{(i)}\right)\right)-U\left(\boldsymbol{R}_{f}\left(\boldsymbol{x}^{(i+1)}\right)\right)<10^{-2} \quad\left(\mathrm{SM}_{\mathrm{TR}}\right.$ and $\mathrm{SM}_{\mathrm{TR}-\mathrm{B}}$ only). 


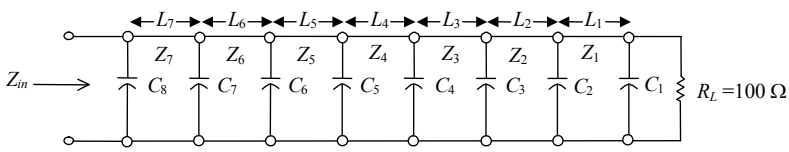

(a)

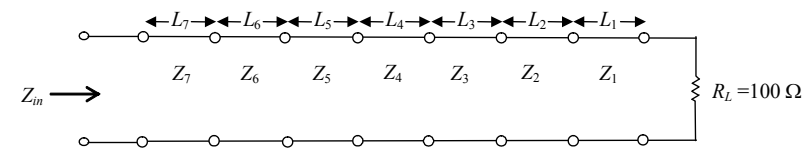

(b)

Fig. 1. Seven-section capacitively-loaded impedance transformer: "fine" model (a) and "coarse" model (b) [10].

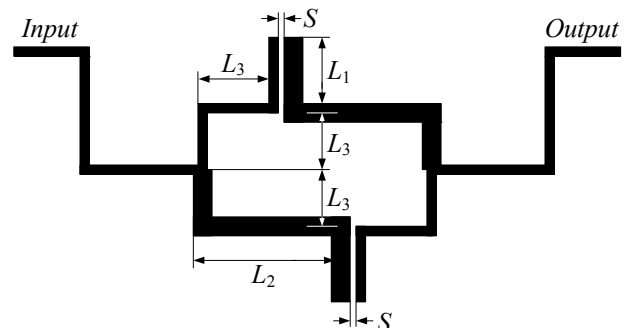

Fig. 2. Geometry of the CCDBR microstrip filter [11].

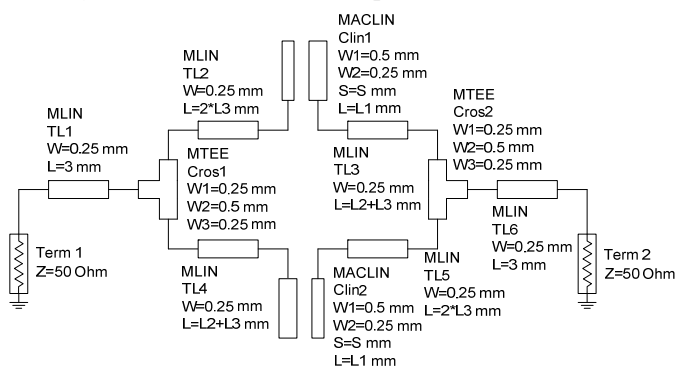

Fig. 3. Coarse model of the CCDBR microstrip filter (Agilent ADS).

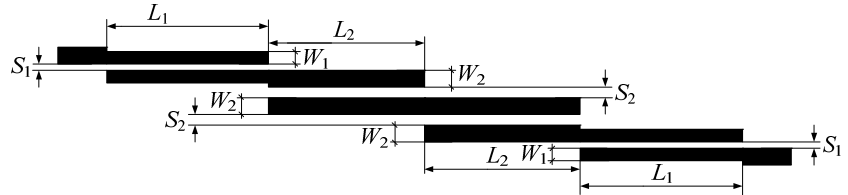

Fig. 4. Third-order Chebyshev bandpass filter [14].

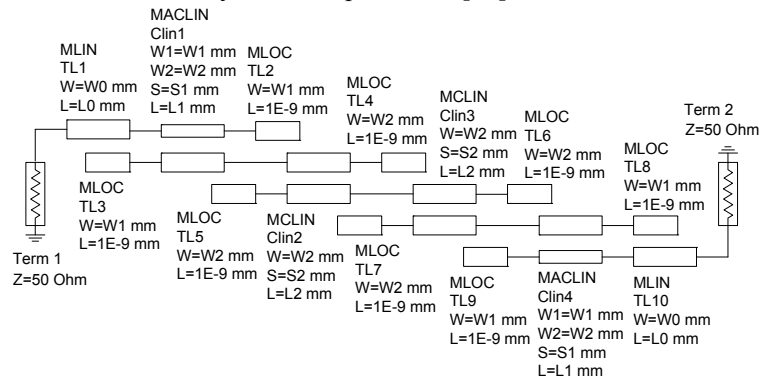

Fig. 5. Coarse model $\boldsymbol{R}_{c}$ of the third-order Chebyshev filter (Agilent ADS).

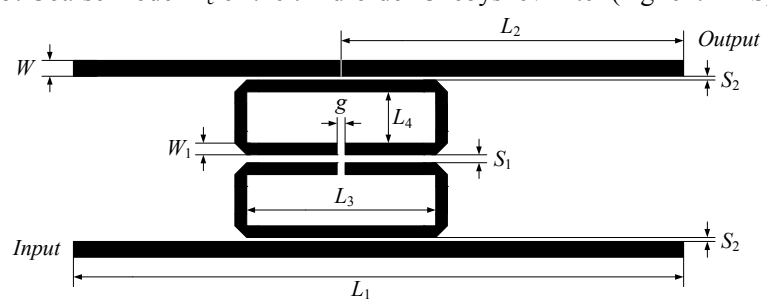

Fig. 6. Open-loop ring resonator bandpass filter [16].

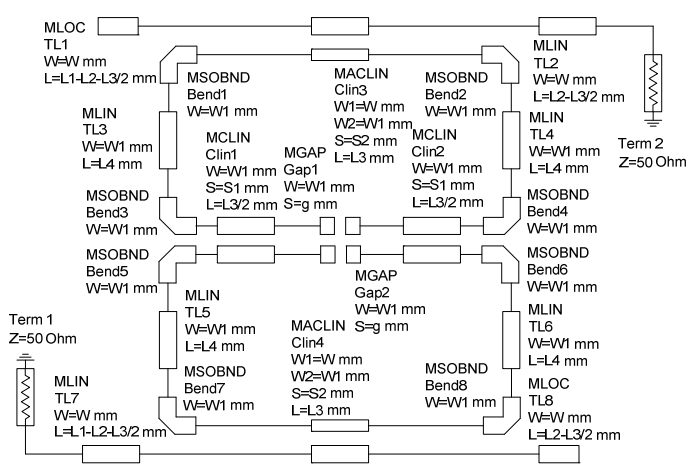

Fig. 7. Coarse model $\boldsymbol{R}_{c}$ of the open-loop ring resonator filter (Agilent ADS).

\section{Results and Discussion}

Table I shows the numerical results. For each problem we tried two different surrogate models. Figures 8-11 show the initial fine model response (corresponding to the coarse model optimal design) and the optimized fine model response for the four problems considered and selected SM algorithms. Figure 12 illustrates the convergence improvement when using algorithm $\mathrm{SM}_{\mathrm{TR}-\mathrm{B}}$ for Problem 1. Figure 13 illustrates the performance amendment when using algorithm $\mathrm{SM}_{\mathrm{TR}-\mathrm{B}}$ for Problem 4.

TABLE I

SM $_{\text {STD }}$ VERSUS SM TR AND SM $_{\text {TR-B }}$ : PERFORMANCE COMPARISON

\begin{tabular}{|c|c|c|c|c|c|}
\hline \multirow[b]{2}{*}{$\begin{array}{c}\text { Test } \\
\text { Problem }\end{array}$} & \multirow[b]{2}{*}{$\overline{\boldsymbol{R}}_{s}$} & \multirow{2}{*}{$\begin{array}{c}\text { SM } \\
\text { Algorithm }\end{array}$} & \multicolumn{2}{|c|}{ Specification Error } & \multirow{2}{*}{$\begin{array}{l}\text { Number of } \\
\text { Fine Model } \\
\text { Evaluations }\end{array}$} \\
\hline & & & Final & $\begin{array}{c}\text { Best } \\
\text { Found }\end{array}$ & \\
\hline \multirow{6}{*}{1} & \multirow{3}{*}{$\boldsymbol{R}_{c}(\boldsymbol{B} \cdot \boldsymbol{x}+\boldsymbol{c})$} & $\mathrm{SM}_{\mathrm{STD}}$ & +0.0271 & -0.0055 & $21^{\#}$ \\
\hline & & $\mathrm{SM}_{\mathrm{TR}}$ & -0.0078 & -0.0078 & 6 \\
\hline & & $\mathrm{SM}_{\mathrm{TR}-\mathrm{B}}$ & -0.0091 & -0.0091 & 7 \\
\hline & \multirow{3}{*}{$\boldsymbol{A} \cdot \boldsymbol{R}_{c}(\boldsymbol{x}+\boldsymbol{c})$} & $\mathrm{SM}_{\mathrm{STD}}$ & -0.0096 & -0.0096 & 6 \\
\hline & & $\mathrm{SM}_{\mathrm{TR}}$ & -0.0096 & -0.0096 & 6 \\
\hline & & $\mathrm{SM}_{\mathrm{TR}-\mathrm{B}}$ & -0.0096 & -0.0096 & 7 \\
\hline \multirow{6}{*}{2} & \multirow{3}{*}{$\boldsymbol{R}_{c}(\boldsymbol{x}, \boldsymbol{F}, \boldsymbol{I})^{*}, \$$} & $\mathrm{SM}_{\mathrm{STD}}$ & -1.4 & -1.4 & 10 \\
\hline & & $\mathrm{SM}_{\mathrm{TR}}$ & -1.5 & -1.5 & 12 \\
\hline & & $\mathrm{SM}_{\mathrm{TR}-\mathrm{B}}$ & -1.6 & -1.6 & 12 \\
\hline & \multirow{3}{*}{$\boldsymbol{R}_{c}(\boldsymbol{x}+\boldsymbol{c})$} & $\mathrm{SM}_{\mathrm{STD}}$ & -2.4 & -2.4 & 12 \\
\hline & & $\mathrm{SM}_{\mathrm{TR}}$ & -2.3 & -2.3 & 11 \\
\hline & & $\mathrm{SM}_{\mathrm{TR}-\mathrm{B}}$ & -2.5 & -2.5 & 11 \\
\hline \multirow{6}{*}{3} & \multirow{3}{*}{$\boldsymbol{R}_{c}(\boldsymbol{x}+\boldsymbol{c})$} & $\mathrm{SM}_{\mathrm{STD}}$ & -0.1 & -0.1 & 8 \\
\hline & & $\mathrm{SM}_{\mathrm{TR}}$ & -0.1 & -0.1 & 8 \\
\hline & & $\mathrm{SM}_{\mathrm{TR}-\mathrm{B}}$ & -1.0 & -1.0 & 10 \\
\hline & \multirow{3}{*}{$\boldsymbol{R}_{c}(\boldsymbol{x}+\boldsymbol{c}, \boldsymbol{F})^{*}$} & $\mathrm{SM}_{\mathrm{STD}}$ & -0.4 & -0.7 & 8 \\
\hline & & $\mathrm{SM}_{\mathrm{TR}}$ & -0.7 & -0.7 & 7 \\
\hline & & $\mathrm{SM}_{\mathrm{TR}-\mathrm{B}}$ & -0.7 & -0.7 & 8 \\
\hline \multirow{6}{*}{4} & \multirow{3}{*}{$\boldsymbol{R}_{c}(\boldsymbol{x}, \boldsymbol{F})^{*}$} & $\mathrm{SM}_{\mathrm{STD}}$ & +0.6 & -0.9 & $21^{\#}$ \\
\hline & & $\mathrm{SM}_{\mathrm{TR}}$ & -1.2 & -1.2 & 11 \\
\hline & & $\mathrm{SM}_{\mathrm{TR}-\mathrm{B}}$ & -1.2 & -1.2 & 11 \\
\hline & \multirow{3}{*}{$\boldsymbol{R}_{c}(\boldsymbol{x}, \boldsymbol{I})^{\$}$} & $\mathrm{SM}_{\mathrm{STD}}$ & -1.8 & -1.8 & 12 \\
\hline & & $\mathrm{SM}_{\mathrm{TR}}$ & -1.8 & -1.8 & 6 \\
\hline & & $\mathrm{SM}_{\mathrm{TR}-\mathrm{B}}$ & -1.8 & -1.8 & 8 \\
\hline
\end{tabular}

${ }^{\#}$ Convergence not obtained; algorithm terminated after 20 iterations.

${ }^{*} \boldsymbol{F}$ denotes frequency SM, where the coarse model is evaluated at frequencies different from the original sweep according to $\omega \rightarrow f_{1}+f_{2} \omega$, with $\boldsymbol{F}=\left[f_{1} f_{2}\right]^{T}$.

${ }^{\$} I$ stands for implicit SM [2] with the substrate height and dielectric constants used to improve the matching between the surrogate and fine models. 
Our results indicate the advantages of $\mathrm{SM}_{\mathrm{TR}}$ and $\mathrm{SM}_{\mathrm{TR}-\mathrm{B}}$ over the standard $\mathrm{SM}$ algorithm. $\mathrm{SM}_{\mathrm{TR}}$ ensures convergence for all considered cases, and improves performance in terms of the specification error corresponding to the optimized design for some cases. Algorithm $\mathrm{SM}_{\mathrm{TR}-\mathrm{B}}$, on the other hand, is able to bring, in some cases, further improvement in terms of the quality of the final design with little or no extra computational overhead. In the cases where the $\mathrm{SM}_{\mathrm{STD}}$ does well, both $\mathrm{SM}_{\mathrm{TR}}$ and $\mathrm{SM}_{\mathrm{TR}-\mathrm{B}}$ have little or no effect, which is exactly what we require.

\section{CONCLUSION}

A systematic treatment of trust-region enhanced SM algorithms is presented. A performance comparison with the standard SM indicates that our trust region approach is a suitable tool to improve the robustness of SM algorithms.

\section{REFERENCES}

[1] J.W. Bandler, Q.S. Cheng, S.A. Dakroury, A.S. Mohamed, M.H. Bakr, K. Madsen, and J. Søndergaard, "Space mapping: the state of the art," IEEE Trans. Microwave Theory Tech., vol. 52, no. 1, pp. 337-361, Jan. 2004.

[2] S. Koziel, J.W. Bandler, and K. Madsen, "A space mapping framework for engineering optimization: theory and implementation," IEEE Trans. Microwave Theory Tech., vol. 54, no. 10, pp. 3721-3730, Oct. 2006.

[3] S. Amari, C. LeDrew, and W. Menzel, "Space-mapping optimization of planar coupled-resonator microwave filters," IEEE Trans. Microwave Theory Tech., vol. 54, no. 5, pp. 2153-2159, May 2006.

[4] S. Koziel and J.W. Bandler, "Space-mapping optimization with adaptive surrogate model," IEEE Trans. Microwave Theory Tech., vol. 55, no. 3, pp. 541-547, March 2007.

[5] A.R. Conn, N.I.M. Gould, and P.L. Toint, Trust Region Methods, MPSSIAM Series on Optimization, 2000

[6] N.M. Alexandrov and R.M. Lewis, "An overview of first-order model management for engineering optimization," Optimization Eng., vol. 2, no. 4, pp. 413-430, Dec. 2001.

[7] N.M. Alexandrov, J. E. Dennis, R. M. Lewis, and V. Torczon, "A trust region framework for managing use of approximation models in optimization," Struct. Multidisciplinary Optim., vol. 15, no. 1, pp. 16-23, 1998.

[8] X. Zhu, A. Hasib, N.K. Nikolova, and M.H. Bakr, "Efficient electromagnetic optimization using self-adjoint Jacobian computation based on a central-node FDFD method," IEEE MTT-S Int. Microwave Symposium, June 2008, pp. 979-982.

[9] C.G. Broyden, "A class of methods for solving nonlinear simultaneous equations," Math. Comput., vol. 19, pp. 577-593, 1965.

[10] M.H. Bakr, J.W. Bandler, K. Madsen, and J. Søndergaard, "An introduction to the space mapping technique," Optimization Eng., vol. 2, no. 4, pp. 369-384, Dec. 2001

[11] A. Manchec, C. Quendo, J.-F. Favennec, E. Rius, and C. Person, "Synthesis of capacitive-coupled dual-behavior resonator (CCDBR) filters," IEEE Trans. Microwave Theory Tech., vol. 54, no. 6, pp. 23462355, June 2006.

[12] FEKO $^{\circledast}$ User's Manual, Suite 5.3, 2008, EM Software \& Systems-S.A. (Pty) Ltd, 32 Techno Lane, Technopark, Stellenbosch, 7600, South Africa.

[13] Agilent ADS, Version 2008, Agilent Technologies, 1400 Fountaingrove Parkway, Santa Rosa, CA 95403-1799, 2008.

[14] J. T. Kuo, S. P. Chen, and M. Jiang, "Parallel-coupled microstrip filters with over-coupled end stages for suppression of spurious responses," IEEE Microwave and Wireless Comp. Lett., vol. 13, no. 10, pp. 440-442, Oct. 2003.

[15] $\boldsymbol{e m}^{\mathrm{TM}}$ Version 11.53, Sonnet Software, Inc., 100 Elwood Davis Road, North Syracuse, NY 13212, USA, 2008.

[16] C.Y. Chen and C.Y. Hsu, "A simple and effective method for microstrip dual-band filters design," IEEE Microwave and Wireless Comp. Lett., vol. 16 , no. 5, pp. 246-248, May 2006.

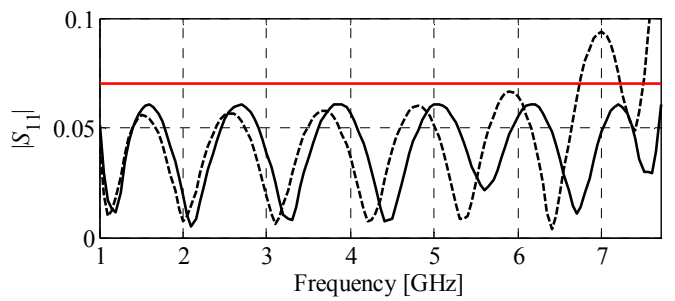

Fig. 8. Problem 1: Initial (dashed line) and optimized (solid line) $\left|S_{21}\right|$ versus frequency; optimization using $\mathrm{SM}_{\mathrm{TR}-\mathrm{B}}$ algorithm with the $\boldsymbol{A} \cdot \boldsymbol{R}_{c}(\boldsymbol{x}+\boldsymbol{c})$ model.

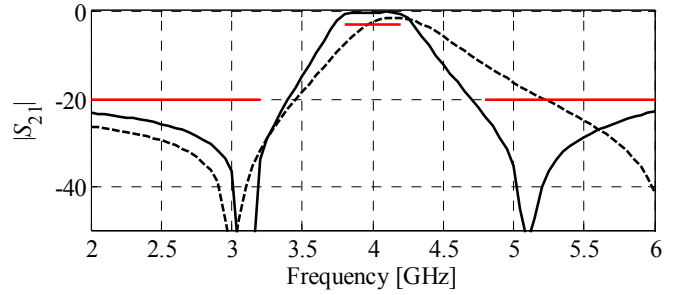

Fig. 9. Problem 2: Initial (dashed line) and optimized (solid line) $\left|S_{21}\right|$ versus frequency; optimization using SM $\mathrm{SM}_{\mathrm{TR}-\mathrm{B}}$ algorithm with the $\boldsymbol{R}_{c}(\boldsymbol{x}+\boldsymbol{c})$ model.

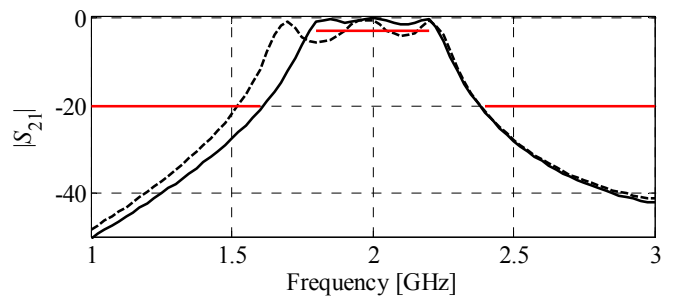

Fig. 10. Problem 3: Initial (dashed line) and optimized (solid line) $\left|S_{21}\right|$ versus frequency; optimization using $\mathrm{SM}_{\mathrm{TR}-\mathrm{B}}$ algorithm with the $\boldsymbol{R}_{c}(\boldsymbol{x}+\boldsymbol{c})$ model.

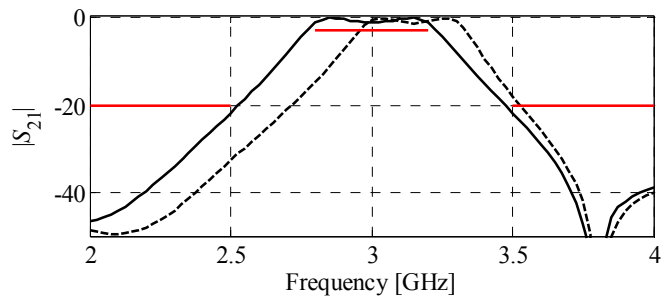

Fig. 11. Problem 4: Initial (dashed line) and optimized (solid line) $\left|S_{21}\right|$ versus frequency; optimization using $\mathrm{SM}_{\text {TR-B }}$ algorithm with the $\boldsymbol{R}_{c}(\boldsymbol{x}, \boldsymbol{I})$ model.

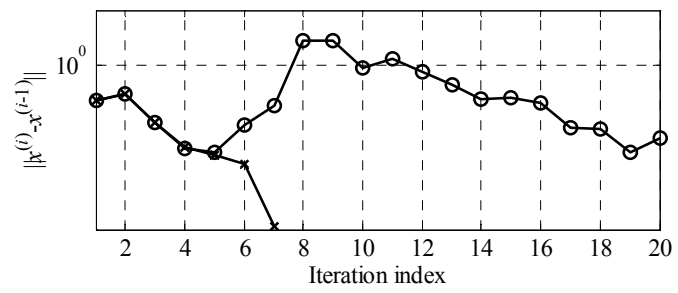

Fig. 12. Problem 1: Convergence plots for $\mathrm{SM}_{\mathrm{STD}}(\mathrm{o})$ and $\mathrm{SM}_{\mathrm{TR}-\mathrm{B}}(\times)$, both using surrogate model $\boldsymbol{R}_{c}(\boldsymbol{B} \cdot \boldsymbol{x}+\boldsymbol{c})$, versus iteration index.

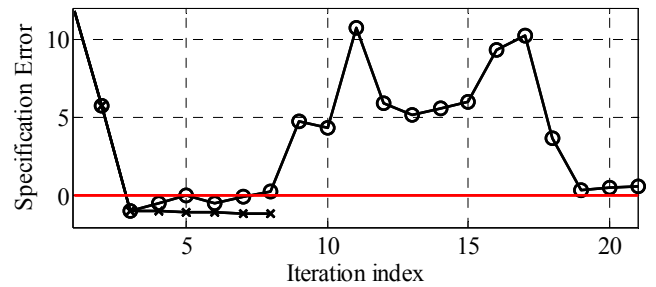

Fig. 13. Problem 4: Evolution of the specification error for $\mathrm{SM}_{\mathrm{STD}}$ (o) and $\mathrm{SM}_{\mathrm{TR}-\mathrm{B}}(\times)$, both using surrogate model $\boldsymbol{R}_{c}(\boldsymbol{x}, \boldsymbol{F})$, versus iteration index. 